Harold A. McAlister and William I. Hartiopf (eds.)

\title{
Miras Without Masers are Symbiotic Stars
}

\author{
B. M. LEWIS \\ Arecibo Observatory, PO Box 995, Arecibo PR 00612, USA
}

\begin{abstract}
About $40 \%$ of potential OH / IR stars, color selected from the IRAS Point Source Catalog, have no $1612 \mathrm{MHz}$ masers. While these objects are rarely carbon rich, they are usually associated with circumstellar shells. The natural explanation for these "OH / IR star color mimics" is that they are systems with a degenerate companion collecting an accretion disk from a red giant wind. This provides them with an extra source of UV for dissociating their molecules. The persistent absence of the usual complement of masers from an O-rich shell is then a pointer to the presence of a degenerate companion. These occur in association with $\sim 45 \%$ of old giant stars.
\end{abstract}

\section{INTRODUCTION}

Radio frequency observers of circumstellar shells have normally thought about miras and $\mathrm{OH}$ / IR stars as solitary stars, despite the existence of well-known binaries, like Mira, and despite the frequency of binaries in general. We now have to change this habit, as $<55 \%$ of the color selected IRAS sources expected to exhibit $1612 \mathrm{MHz}$ masers have them. Lewis (1992) shows that this result is common to all observers, is not an artifact of deployed sensitivity, and is not a result of source variability. Nor is it due to these objects being carbon-rich, as most with low resolution IRAS spectra exhibit a $9.7 \mu \mathrm{m}$ silicate feature, and some even exhibit water or mainline $\mathrm{OH}$ masers, though not a $1612 \mathrm{MHz}$ maser. This phenomenology was initially surprising, as "robust" $1612 \mathrm{MHz}$ masers only require a flux of 35 and $53 \mu \mathrm{m}$ pump photons and a suitable column density of $\mathrm{OH}$ molecules to exist: these needs are implicitly met by sources with the selected IR colors, unless they are binaries.

Our puzzle is completely solved if each of these "OH / IR star color mimics" has a degenerate companion. Since this sits in a red giant's wind, it collects an accretion disk, and becomes a potent local source of UV. This UV will destroy molecules within the dust shell before they are accessible to interstellar UV, which weakens or suppresses any masers. The intensity of the extra UV flux depends on the separation of the companion, the mass-loss rate from the red giant, and the extent to which the white dwarf has cooled. So the efficiency of molecule destruction varies between objects. The symbiotic novae provide the most extreme examples of this scenario, as they are strong UV and X-ray sources, and have no masers. On the other hand Mira is near the opposite extreme with its weak masers, low mass-loss rate and a known, cool, distant white dwarf companion, which IUE sees as a $\mathrm{T} \approx 11000 \mathrm{~K}$ accretion disk.

This scenario is recognizably the same as that for $\mathrm{D}$ (usty)-type symbiotic stars (e.g., Allen 1988). Symbiotics normally have two different temperature regimes reflected in their optical spectra; D-types are composed of a mass-losing red giant interacting with a white dwarf companion, that lies beyond the radius for Roche Lobe interactions. In the case of OH / IR stars, the circumstellar shell is optically thick, which obviates direct inspection of their spectra. But a generalized D-type symbiotic system can still be diagnosed, since a red giant 
is always responsible for the easily observed and characteristic IR signal from the dust shell, while the absence of the usual complement of masers flags the presence of molecule destroying, "high temperature" UV.

An independent feature of an accretion disk is the extra energy it dissipates in the surrounding dust shell. Since dust in its vicinity is hotter than it would otherwise be, it radiates at a higher temperature. This causes the integrated IR colors of the shell to be displaced to the blue when the disk is not occulted. The prototype for this effect is R Aqr (Whitelock et al. 1987). A blue displacement is shown by $24 \%$ of objects with low resolution spectra (LRS) types 38-39. These are particularly distinctive, as they have the largest mass-loss rates and show the $9.7 \mu \mathrm{m}$ feature most strongly in absorption.

\section{WHITE DWARF FREQUENCIES}

Since $>40 \%$ of color selected IR sources are without masers, this scenario implies a high corresponding frequency of degenerate (usually white dwarf) companions. Normal masing shells should arise whenever (i) the companion is a reasonably cool main sequence star, that generates little UV; (ii) when the companion is a common envelope object at the mira phase; (iii) when the mass-losing red giant is intrinsically solitary. The mira phase may perhaps be aborted or modified if the companion is at an intermediate distance that allows for Roche Lobe interactions. But widely spaced degenerate companions permit a mira its normal development. These collect some mass from its outflowing wind and decimate some of its molecules. The molecular content of the wind is thus a sensitive probe for the presence of a degenerate companion (DC).

The percentage of DCs in giant binaries is enhanced above the 50:50 rate assumed for an arbitrary inspection epoch during a Hubble time, by (i) the fact that the more massive star evolves more quickly, and so is available for inspection as a red giant for a shorter interval; and (ii) the tendency for the proportion of widely separated systems (WSS) to be augmented by those with expanding separations as a primary loses mass during its mira phase. We can use the $\approx 55 \%$ frequency of IR sources with $1612 \mathrm{MHz}$ masers in color selected samples, together with Eggleton's (1990) limit of $\geq 30 \%$ for the number of close companions among giant $\mathrm{K}$ stars, to estimate a lower limit to the number of DCs to expect from widely separated systems of $R \sim 45 /\{100-30\} \geq 64 \%$.

This estimate for $\mathbf{R}$ is increased by the removal of its systematic biases. Thus the denominator is reduced by the percentage of solitary stars, and by any increase in the proportion of close companions above $30 \%$. The numerator is also increased by any allowance for the number of systems with DCs that still have $1612 \mathrm{MHz}$ masers, and some are known. Further more, our estimate for the number of systems without masers is reduced by those with large blue displacements in their IR colors, that are effectively displaced out of an IR color delineated sample. Modest $5 \%$ allowances for each of the first two biases would raise the percentage of WSS with DCs to $77 \%$.

Rather similar results are obtained when we consider the 42 IRAS sources with LRS types 38-39, from stars with progenitor masses of $\sim 2-5 \mathcal{M}_{\odot}$. These are plotted on an IR two-color diagram in Figure 1; 10 are displaced to the blue (left) from their normally expected location, while the remainder overlie 


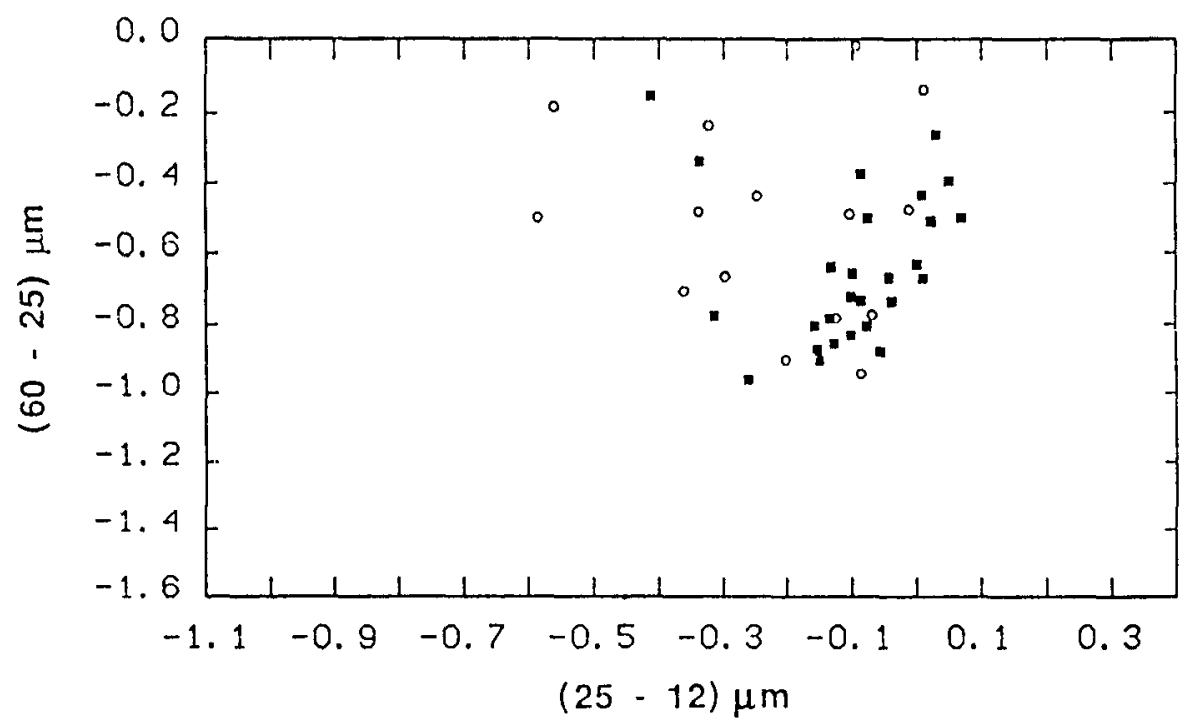

FIGURE 1. The IR two-color diagram of LRS type 38-39 objects, where most massive $\mathrm{OH}$ / IR stars lie. The filled symbols show detected $1612 \mathrm{MHz}$ masers.

the region of the diagram occupied by the most massive OH / IR stars. This sample allows us to identify all of the color displaced objects, and the 8 sources with normal colors that are without $1612 \mathrm{MHz}$ masers, as those we expect to have DCs. The $(10+8)$ DCs are $43 \%$ of the total sample of 42 , which is like the $\sim 45 \%$ of all color selected sources without masers, while the percentage of WSS with DCs, following the first approach above, is $\mathrm{R} \sim 18 /\left\{0.7^{*} 42\right\} \geq 61 \%$.

This approach provides us with a fresh way of showing that most stars with progenitor masses $\geq 0.8 \mathcal{M}_{\odot}$ have companions. The low limit to this estimate comes from amalgamating the $45 \%$ of mimics with Eggleton's $30 \%$ of $\mathrm{K}$ giants with close companions, to get a low $75 \%$ of all systems. This estimate does not include the percentage of WSS with main sequence companions. Clearly searches for planets and SETI searches are best concentrated on objects which show no UV signatures beyond those expected of a visible main sequence star.

This work is supported by NAIC, which is operated by Cornell University under a cooperative management agreement with NSF.

\section{REFERENCES}

Allen, D. 1988, The Symbiotic Phenomenon, ed J. Mikolajewska, M. Friedjung, S.J. Kenyon, and R. Viotti, ASSL 145, (Dordrecht, Kluwer), p. 3 Eggleton, P. P. 1990, From Miras to PN: Which Path for Stellar Evolution, p 513 Joint IRAS Working Group, 1985, IRAS Point Source Catalog, (Washington, DC, U.S. GPO)

Lewis, B.M. 1992, ApJ, in press (Sep 1)

Whitelock, P.A., Pottasch, S.R., \& Feast, M.W. 1987, in Late Stages in Stellar

Evolution, eds. S. Kwok and S. R. Pottasch, (Dordrecht, Reidel), p. 269 